\title{
Review of high-contrast imaging systems for current and future ground-based and space-based telescopes II. Common path wavefront sensing/control and Coherent Differential Imaging
}

Nemanja Jovanovic $^{\mathrm{a}}$, Olivier Absil ${ }^{\mathrm{b}}$, Pierre Baudoz ${ }^{\mathrm{c}}$, Mathilde Beaulieu ${ }^{\mathrm{d}}$, Michael Bottom ${ }^{\mathrm{e}}$, Eric Cady ${ }^{\mathrm{e}}$, Brunella Carlomagno ${ }^{\mathrm{b}}$, Alexis Carlotti ${ }^{\mathrm{f}}$, David Doelman ${ }^{\mathrm{g}}$, Kevin Fogarty ${ }^{\mathrm{h}}$, Raphaël Galicher ${ }^{\mathrm{c}}$, Olivier Guyon ${ }^{\mathrm{i}, \mathrm{j}, \mathrm{k}}$, Sebastiaan Haffert ${ }^{\mathrm{g}}$, Elsa Huby ${ }^{\mathrm{c}}$, Jeffrey Jewell ${ }^{\mathrm{e}}$, Christoph Kellerg, Matthew A. Kenworthy ${ }^{\mathrm{g}}$, Justin Knight ${ }^{\mathrm{j}, 1}$, Jonas Kühn ${ }^{\mathrm{m}}$, Kelsey Miller ${ }^{\mathrm{j}, 1}$, Johan Mazoyer ${ }^{h, n}$, Mamadou N'Diaye ${ }^{\mathrm{d}}$, Emiel Por ${ }^{\mathrm{g}}$, Laurent Pueyo ${ }^{\mathrm{h}}$, A J Eldorado Riggs ${ }^{\mathrm{e}}$, Garreth Ruane $^{\mathrm{a}}$, Dan Sirbu ${ }^{\mathrm{o}}$, Frans Snik ${ }^{\mathrm{g}}$, J. Kent Wallace ${ }^{\mathrm{e}}$, Michael Wilby ${ }^{\mathrm{g}}$, and Marie Ygouf ${ }^{\mathrm{p}}$

${ }^{a}$ California Institute of Technology, 1200 E. California Blvd., Pasadena, CA 91125, USA

${ }^{\mathrm{b}}$ Space sciences, Technologies, and Astrophysics Research (STAR) Institute, University of Liège, 19C allée du Six Août, B-4000 Liège, Belgium

${ }^{\mathrm{c}}$ LESIA, Observatoire de Paris, PSL Research University, CNRS, Sorbonne Universités, Univ.

Paris Diderot, UPMC Univ. Paris 06, Sorbonne Paris Cité, 5 place Jules Janssen, 92190 Meudon, France

'Université Cote d'Azur, Observatoire de la Cote d'Azur, CNRS, Laboratoire Lagrange, Parc Valrose, F-06108 Nice, France

e Jet Propulsion Laboratory, California Institute of Technology, 4800 Oak Grove Drive, Pasadena, CA 91109 USA

${ }^{\mathrm{f}} \mathrm{CNRS}$, Institut de Plantologie et d'Astrophysique de Grenoble (IPAG), F-38000, Grenoble, France

'Leiden Observatory, Leiden University, P.O. Box 9513, 2300 RA Leiden, The Netherlands

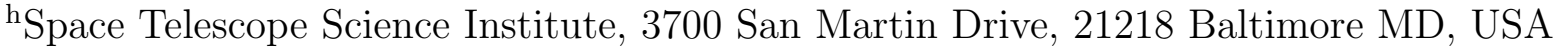

iAstrobiology Center, National Institutes of Natural Sciences, 2-21-1 Osawa, Mitaka, Tokyo, JAPAN

jSteward Observatory, University of Arizona, Tucson, AZ 85721, USA

${ }^{k}$ National Astronomical Observatory of Japan, Subaru Telescope, National Institutes of Natural Sciences, Hilo, HI 96720, USA

${ }^{1}$ College of Optical Sciences, University of Arizona, 1630 E University Blvd, Tucson, AZ 85719, USA

${ }^{m}$ Institute for Particle Physics and Astrophysics, ETH Zurich, Wolfgang-Pauli-Str. 27, CH-8093 Zurich, Switzerland

${ }^{n}$ Johns Hopkins University, Zanvyl Krieger School of Arts and Sciences, Department of Physics and Astronomy, Bloomberg Center for Physics and Astronomy, 3400 North Charles Street, Baltimore, MD 21218, USA

${ }^{\circ}$ NASA Ames Research Center, Moffett Field, Mountain View, CA, 94035, USA PIPAC, Caltech, 1200 E. California Blvd., Pasadena, CA 91125, USA

Further author information: (Send correspondence to Nemanja Jovanovic)

Nemanja Jovanovic: E-mail: jovanovic.nem@gmail.com, Telephone: +1 6263951214 


\begin{abstract}
The Optimal Optical Coronagraph (OOC) Workshop held at the Lorentz Center in September 2017 in Leiden, the Netherlands, gathered a diverse group of 25 researchers working on exoplanet instrumentation to stimulate the emergence and sharing of new ideas. In this second installment of a series of three papers summarizing the outcomes of the OOC workshop (see also 1,2), we present an overview of common path wavefront sensing/control and Coherent Differential Imaging techniques, highlight the latest results, and expose their relative strengths and weaknesses. We layout critical milestones for the field with the aim of enhancing future ground/space based high contrast imaging platforms. Techniques like these will help to bridge the daunting contrast gap required to image a terrestrial planet in the zone where it can retain liquid water, in reflected light around a $\mathrm{G}$ type star from space.
\end{abstract}

Keywords: Wavefront sensing, common path wavefront sensing, coherent differential imaging, high contrast imaging, exoplanets

\title{
1. INTRODUCTION
}

While conventional wavefront sensors (WFSs) measure aberrations using light extracted ahead of the coronagraph optics (usually by a beam splitter), common-path WFSs rely on either exploiting the post-coronagraph focal plane image or the light diffracted by the focal plane mask to infer wavefront aberrations up to the coronagraphic focal plane. In this case the science camera is most commonly used for wavefront sensing. The approach offers two significant advantages: (1) it is free of optical non-common path errors and the measurement is performed at the exact same wavelength as science acquisition and (2) it is highly sensitive, efficiently converting wavefront errors into intensity modulation.

One challenge is that it is not straightforward to recover wavefront measurements from focal plane intensity alone. Many approaches have been demonstrated to break this degeneracy - all of them relying on coherent mixing with the bright on-axis starlight (see Table 1). The techniques fall into two major categories, namely those that utilize spatial mixing and those that rely on temporal mixing. Spatial mixing refers to techniques that rely on either the continuous interference between the speckle field and a deliberate and permanent probe (e.g. Modal wavefront sensor, self-coherent camera) or the correlation between various points in the complex field about the point-spread function (PSF) (e.g. linear dark field control, Kernel phase). On the other hand, temporal modulation requires the injection of known perturbations, typically with a deformable mirror (DM) or a tip/tilt mirror and the subsequent tracking of the response of the system as a function of time (speckle nulling, pairwise probing, COFFEE, MEDUSAE, phase retrieval). Owing to the fact that some of the science images collected are during probing, the duty cycle of the class of temporal mixing techniques is $<100 \%$.

As originally proposed and implemented, the goal of common-path WFSs was solely to measure coherent light in the focal plane, so that a control loop could drive this residual light (coronagraph leakage) toward zero (usually by DM actuation). The same signals that lead to wavefront measurements can also be processed to decompose the focal plane image into both a coherent component and an incoherent component. The coherent measurement drives the high contrast control loop, while the incoherent component contains the astrophysical signal, and should therefore be collected. The approach of modulating the speckle field to determine the incoherent/coherent parts of the field is referred to as coherent differential imaging (CDI) and is typically undertaken in post-processing (similar to angular differential imaging or spectral differential imaging).

Owing to the finite temporal bandwidth of the wavefront control loop versus the coherence time of the atmosphere, the limited sensitivity of a WFS, detector noise, chromatic errors and so on, real time correction using a common-path WFS can only improve the contrast over a conventional WFS by so much. Post-processing via CDI methods will be critical to enhance the contrast beyond these limitations and bridge the extreme contrast of $10^{10}$ between a $\mathrm{G}$ type star like our sun and a terrestrial planet orbiting it in the habitable zone.

In this paper we review some of the common-path WFSing/CDI techniques and outline what has been achieved to date. We list some lessons learned and set out milestones for the field to be used as guidelines for development to enable imaging of terrestrial exoplanets in the future. 
Table 1. A list of the various common-path WFSing techniques. LDFC - Linear dark field control, MEDUSAE - Multispectral Exoplanet Detection Using Simultaneous Aberration Estimation, QACITS - Quadrant Analysis of Coronagraphic Images for Tip-tilt Sensing, SCC - Self coherent camera, COFFEE - COronagraphic Focal-plane wave-Front Estimation for Exoplanet detection.

\begin{tabular}{|c|c|c|c|c|}
\hline Technique & Modulation used & Real time & Science duty cycle & Section \\
\hline Modal WFS 3 & no & yes & $100 \%$ & 2.1 \\
\hline LDFC 4,5 & no & yes & $100 \%$ & 2.2 \\
\hline MEDUSAE 6 & no & no & $<100 \%$ & 2.3 \\
\hline COFFEE 7 & yes & no & $<100 \%$ & 2.3 \\
\hline QACITS 8,9 & no & yes & $100 \%$ & 2.4 \\
\hline SCC 10 & yes & yes & $100 \%$ & 2.5 \\
\hline Pairwise probing 11 & yes & no & $<100 \%$ & 2.6 \\
\hline Speckle nulling 12,13 & yes & yes & $<100 \%$ & 2.7 \\
\hline Phase retrieval 14 & yes & no & $<100 \%$ & 2.8 \\
\hline Kernel phase 15,16 & yes & yes & $<100 \%$ & 2.9 \\
\hline Phase shifting interferometry 17 & no & yes & $100 \%$ & 2.10 \\
\hline Phase sorting interferometry 18,19 & no & yes & $100 \%$ & 2.11 \\
\hline
\end{tabular}

\section{OVERVIEW OF COMMON-PATH WAVEFRONT SENSING TECHNIQUES AND STATE OF THE FIELD}

This section steps through a selection of promising common-path WFSing techniques, outlines the basic concept behind each and provides an overview of their maturity and success thus far.

\subsection{Coronagraphic Modal Wavefront Sensor (cMWS)}

In addition to creating dark holes in the stellar PSF, pupil-plane coronagraphs can also be used to encode wavefront-sensing information directly in the science focal plane. In the case of an Apodizing Phase Plate (APP) coronagraph 20,21, phase-only holograms can be multiplexed with the APP pattern to produce pairs of modified copies of the central PSF at arbitrary locations in the focal plane, of arbitrary relative power, and containing independent wavefront biases with respect to the central science image.

The coronagraphic Modal Wavefront Sensor (cMWS) uses this holographic technique to create two oppositely biased PSF copies for each wavefront mode to be sensed 3 (Fig. 1, left panel). The normalized difference between the core intensities of corresponding PSF copies is then directly related to the mode amplitude, allowing fast retrieval of the wavefront coefficients at the focal plane using two intensity measurements per mode.

The creation of each holographic PSF copy requires a small reduction to science throughput, which for the cMWS is typically chosen to be $1 \%$ per mode. This, coupled with limitations on focal-plane detector space, typically makes it impractical to sense more than 20-30 modes. However, a free choice of (orthogonal) sensing mode basis means that the cMWS is not necessarily limited to only low-order aberrations: it is equally possible to use a tailored basis encompassing all spatial frequencies which correspond to a small dark hole a few square $\lambda / \mathrm{D}$ in size.

A cMWS prototype was first implemented on-sky at the William Herschel telescope (WHT) in 2015 3, with improved designs included as an open-loop subsystem of the LEXI instrument with first light in June 2016 23,24. These first tests demonstrated that the cMWS is capable of the real-time sensing of low-order Zernike aberrations injected via reference offsets on the closed-loop AO system, and is able to operate over arbitrarily wide bandwidths (Fig. 1, middle panel). Most recently, successful on-sky closed-loop operation was achieved during the second 

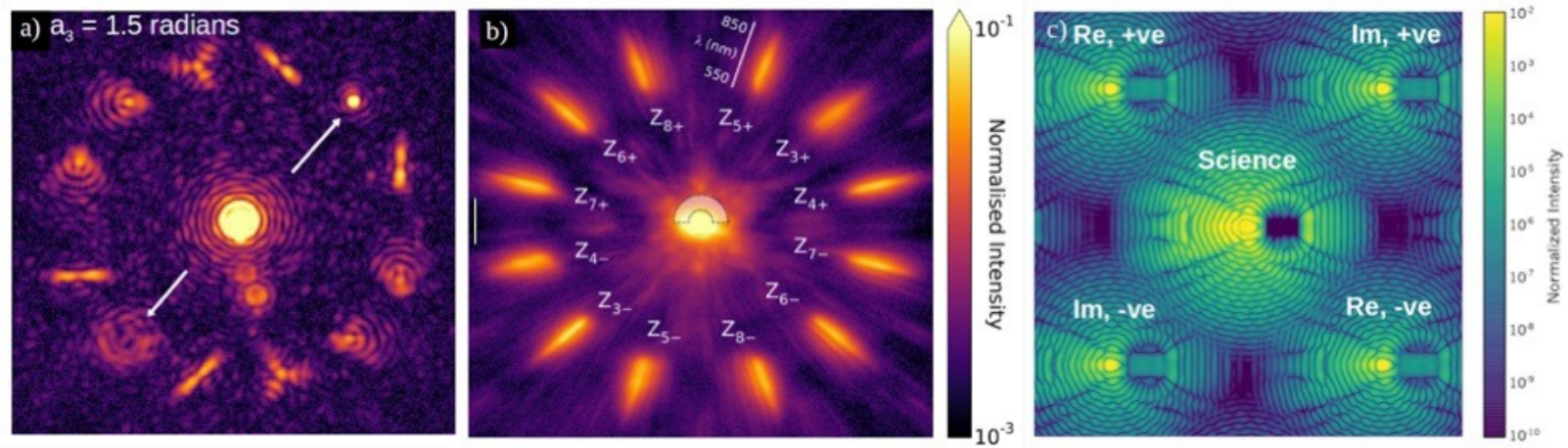

Figure 1. (Left) Laboratory image of a 6-mode Zernike cMWS with 1.5 radians RMS of focus error, showing asymmetrical response of the corresponding focus PSF copies. (Middle) On-sky demonstration of a cMWS at the William Herschel telescope using the same sensing modes, for $20 \%$ bandwidth. (Right) Numerical simulation of a PSF for holographic electric field conjugation, with four copies each containing a different electric field probe in the APP dark hole (Figures adapted from 3,22).

LEXI observing run at the WHT in November 2017 25, using optimized cMWS designs manufactured as liquid crystal phase plates.

This holographic spatial multiplexing approach can also be extended to operate a variety of temporallymodulated focal-plane WFS techniques in a spatially-modulated manner, by using one or more secondary PSF copies instead of the science PSF. This allows for a 100\% duty cycle at the cost of some science throughput, making real-time operation feasible. For example, phase diversity or curvature sensing can be performed by adding a parabolic phase term to a single PSF copy, while electric field conjugation can be achieved by adding well-defined electric field probes to the dark hole of four or more PSF copies 22 (Fig. 1, right panel).

\subsection{Linear Dark Field Control (LDFC)}

Linear dark field control (LDFC) provides a relative measurement of the change in the field based on the change in the intensity of bright speckles outside the dark hole so that the contrast in the dark hole can be maintained. It is not an absolute wavefront measurement technique and so can not be used to dig the dark hole in the first instance.

LDFC relies on two key properties, namely the linear response of the intensity of a bright speckle as a function of the amplitude of the input wavefront perturbation and that there are a subset of aberrations that will effect both the bright field and light in the dark hole simultaneously. Therefore, by examining only the bright speckle field, the contrast in a dark hole next to the PSF core can be controlled/maintained. In this way, aberrations that effect the dark hole can be sensed and canceled, which maintains the dark hole at its initial deep contrast state.

LDFC comes in two flavors: spatial 5 and spectral 4. Spatial LDFC, shown in Fig. 2, uses information from speckles located spatially outside of the dark hole at the science wavelength(s) whereas spectral LDFC senses speckles that occur spatially within the dark hole but spectrally outside of the control bandwidth. In principle, the two can be run simultaneously to compensate for each techniques null space. LDFC can be deployed as a deep contrast stabilization technique for any single-sided dark hole; it cannot be implemented on $360^{\circ}$ dark holes since it relies on access to the same spatial frequencies in the bright field opposite the dark hole. Since it does not rely on modulation or light from the very faint dark hole, LDFC can return the dark hole to its initial deep contrast much faster than modulation-based techniques that probe the field within the dark hole.

Thus far both spatial and spectral LDFC have been simulated and shown to work in principle. While spectral LDFC has not been tested experimentally to date, spatial LDFC was recently demonstrated on the SCExAO instrument (Guyon et al. in prep.). In this demonstration a dark hole was created through speckle nulling (discussed in section 2.7, and then artificial speckles were injected into the hole and shown to be suppressed by the LDFC loop. There are also plans to validate the technique on the University of Arizonas Wavefront Control 


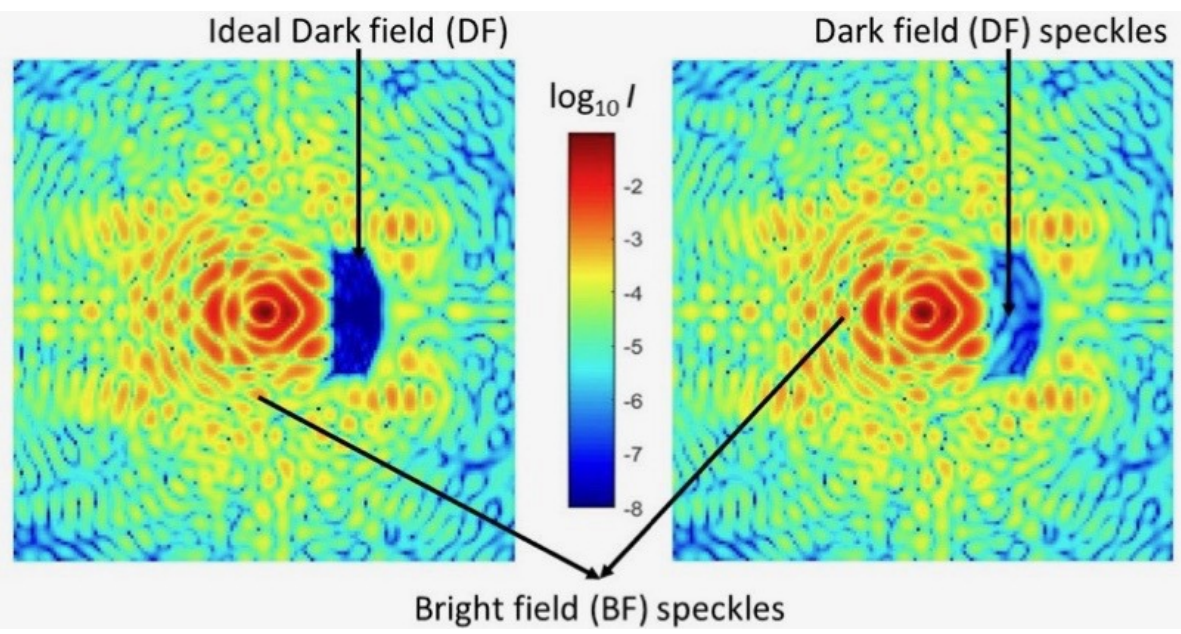

Figure 2. (Left) Spatial LDFC: $3.5 \lambda / D$ x $8.5 \lambda / D$ dark hole/dark field (DF) created in numerical simulation using conventional electric field conjugation (EFC). (Right) Wavefront aberrations produce speckles in the bright field (BF) and the DF which degrade the DF. The change in intensity between the aberrated BF (Right) and the ideal BF (Left) is used to measure and cancel the speckles in the DF.

Testbed with the two single-sided dark holes created by a vector APP coronagraph. Experiments are planned to implement spectral LDFC in a slow, closed-loop fashion using data from the CHARIS IFS 26 behind the SCExAO instrument 27.

\subsection{MEDUSAE/COFFEE}

The MEDUSAE and COFFEE algorithms are two flavors of a family of techniques based on the use of a model of a coronagraphic imaging system that links the speckle field in the focal plane to some parameters of interest (see Fig. 3 for details). These parameters are then estimated using an inverse problem approach in a Bayesian framework. The two techniques have been developed to be complementary and eventually used in synergy (COFFEE for calibration and MEDUSAE for post-processing).

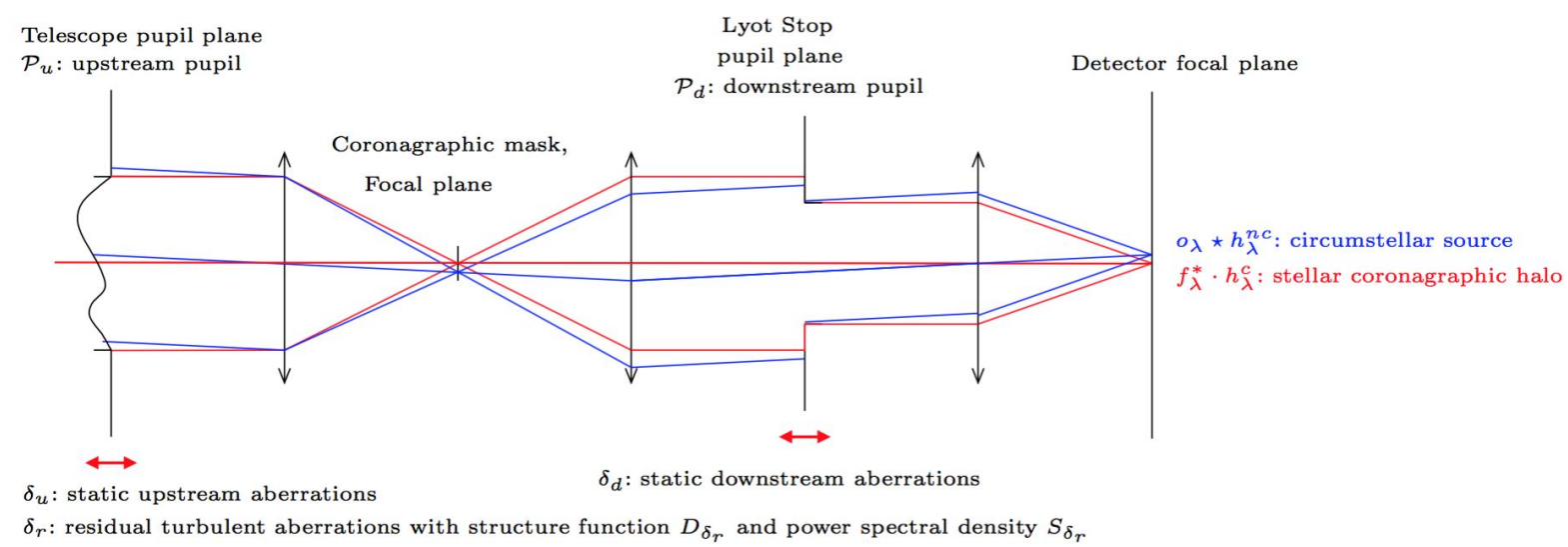

Figure 3. Optical scheme of the coronagraphic imager model used in the MEDUSAE and COFFEE algorithms (Fig. 2 of 6 adapted from 28). In its most current implementation, MEDUSAE estimates jointly the quasi-static instrumental aberrations located upstream of the coronagraph $\delta_{u}$ and the circumstellar source $O_{\lambda}$ directly from multispectral science images. In the most current implementation, COFFEE jointly estimates the upstream electric field and the quasi-static instrumental aberrations downstream $\delta_{d}$ of the coronagraph using DM probes and a realistic model of a coronagraph. 
The MEDUSAE (Multispectral Exoplanet Detection Using Simultaneous Aberration Estimation) postprocessing technique 6 jointly estimates the instrumental aberrations and the object of interest, i.e. the circumstellar environment, in order to separate these two contributions. To do so, it makes use of the Simultaneous Differential Imaging (SDI) strategy by exploiting the wavelength diversity offered by an integral field unit or a dual band imager. The inversion algorithm is based on a maximum a posteriori estimator, which measures a regularized discrepancy between the multispectral data and the imaging model. It is then possible to estimate the aberration map and the circumstellar environment at each wavelength and thus their spectra directly from the science image. Despite the fact that MEDUSAE has been tested and validated on simulated SPHERE-IFS images for the purpose of post-processing 6 , it has the potential to be used in real time in the context of wavefront sensing and control as well. Near-term prospects are to: 1) validate the technique on SPHERE data for post-processing 29 and 2) implement a non-coronagraphic model in order to validate it on the NIRSpec IFU on JWST for post-processing 30 .

COFFEE (COronagraphic Focal-plane wave-Front Estimation for Exoplanet detection) 7 is a coronagraphic phase diversity technique that estimates the instrumental aberrations within a calibration framework. It uses the same model and core routines as MEDUSAE with a few differences in the way it is implemented. For example, it utilizes DM probes for diversity rather than the wavelength diversity used by MEDUSAE. Also, it makes use of a Matrix Fourier Transform (MTF) 31 to simulate a more realistic coronagraph, which is necessary for its implementation on a testbed. The COFFEE principle was demonstrated in the laboratory in Marseille in France on the MITHIC testbed. It was used to estimate the phase aberrations upstream and downstream of a focal plane mask coronagraph from the science image. The minimization of these phase aberrations thanks to a DM led to a $3 \times 10^{-8}$ dark hole at $5 \lambda / D$ separation 32 . Future prospects are to test and validate the technique on several other testbeds including the THD testbed.

\subsection{Quadrant Analysis of Coronagraphic Images for Tip-tilt Sensing (QACITS)}

The QACITS is a tip-tilt sensing technique based on the analysis of the flux asymmetry of the coronagraphic image that appears when the star image is not perfectly centered on the coronagraphic focal plane mask, as shown in Fig.4. This technique is designed to work only with a focal-plane phase mask upstream. The measured differential flux intensity (like in a quad-cell position sensor) within the central area $(<3 \lambda / D)$ is indeed related to the amplitude of the pointing error affecting the beam incident on the focal plane phase mask. Models have been derived for the four quadrant phase mask 8 and for the vortex phase mask 9 . In case of an obstructed pupil, the image analysis has to be done in separate areas in order to disentangle the contribution of light diffracted by the central obstruction 33: the flux is measured in more than four areas.
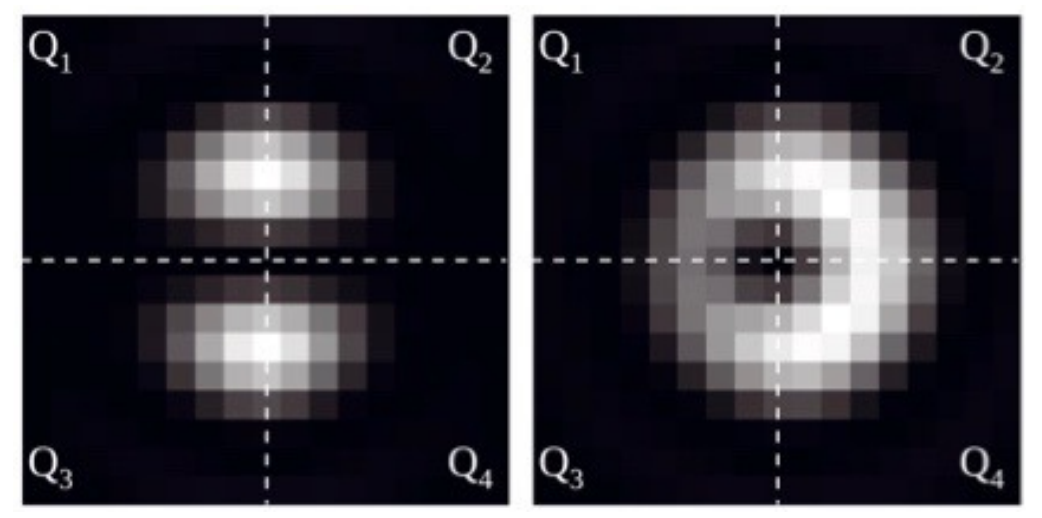

Figure 4. Quadrant analysis of the coronagraphic image in the case of a non obstructed pupil (left) for the four quadrant phase phase mask and (right) for the vortex phase mask. The tip-tilt amplitude is $0.2 \lambda / D$ and the image width is $4 \lambda / D$.

The QACITS algorithm is routinely used on-sky 33 with the vortex coronagraph on the Keck/NIRC2 infrared camera ( $\mathrm{L}$ and $\mathrm{M}$ bands). The control loop is typically run at a frequency of $0.02 \mathrm{~Hz}$ to correct for slow drifts and stabilize the position of the star image behind the coronagraphic focal plane mask down to 2.4 mas RMS. 


\subsection{Self Coherent Camera (SCC)}

The self-coherent camera (SCC) concept utilizes a small off-axis reference hole in the otherwise opaque part of a Lyot stop of a coronagraph, to transmit some of the light rejected by the coronagraph. The downstream optics must be sufficiently large to capture both the primary coronagraphic leakage inside the geometric pupil and the reference beam. By forming an overlapping image with the two beams, Fizeau interference fringes are created across the speckle field in the science image as seen in Fig. 5. In this way, the electric field in the science image is

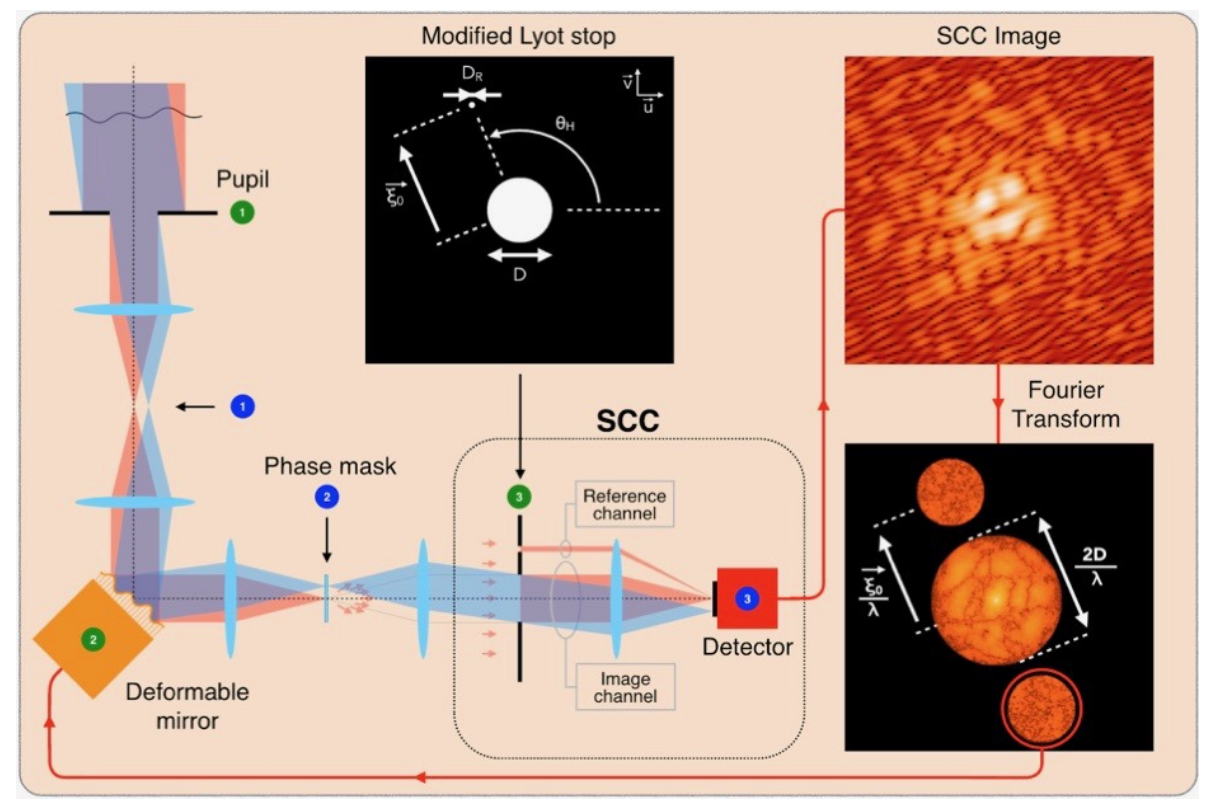

Figure 5. The principle of the SCC. The stellar light (red beam) hits a DM and is focused on a coronagraphic focal plane mask. Most of the light is diffracted outside the geometrical pupil downstream and is blocked by a Lyot stop. The coronagraphic leak is transmitted at the Lyot stop and creates a speckle image on the detector. The small hole carefully positioned in the Lyot stop selects the reference beam which is interfered with the light leaked by the coronagraph which spatially encodes speckles (top right panel). It is then possible to retrieve the speckle electric field using a Fourier transform (bottom right panel).

spatially modulated and can be directly retrieved. Only one image of the science channel is therefore necessary to encode the speckles and estimate the electric field that is to be minimized to create a dark hole: the correction is done at the rate of the science image recording. One can add other reference holes in the Lyot pupil to make the SCC work in polychromatic light 34.

The SCC images can be used for both CDI 10,35, 36 as well as for focal plane wavefront sensing 36-43. Wavefront sensing with the SCC has been demonstrated in both numerical simulations and in the laboratory. It was used to reach a contrast at the $10^{-8}$ level routinely on the THD bench (space-like conditions but no vacuum). It was tested with numerous coronagraph architectures including the four quadrant phase mask 44,45 , the multi four quadrant phase mask 46 , the apodized dual zone phase mask 34,47, the eight-octant phase mask, the vector vortex, and the six level phase mask 48. Results in monochromatic light 49 and $12.5 \%$ bandwidth 47 have been obtained on Meudon THD testbed and both reached a contrast level of $10^{-8}$ before any CDI calibration (see Fig. 6).

Recently, the SCC was also used to control two DMs in cascade in order to correct both phase and amplitude aberrations, resulting in a $360^{\circ}$ dark hole with a $<10^{-8}$ contrast level (figure 7) 50. Finally, the SCC is currently installed at the Palomar Observatory on the stellar double coronagraph instrument for on-sky testing. Gerard et al. (2018) recently proposed a modified focal plane mask for the SCC where fringes would be detected in millisecond-timescale exposures, allowing focal plane wavefront sensing and control of both atmospheric and quasi-static speckles 51 . 

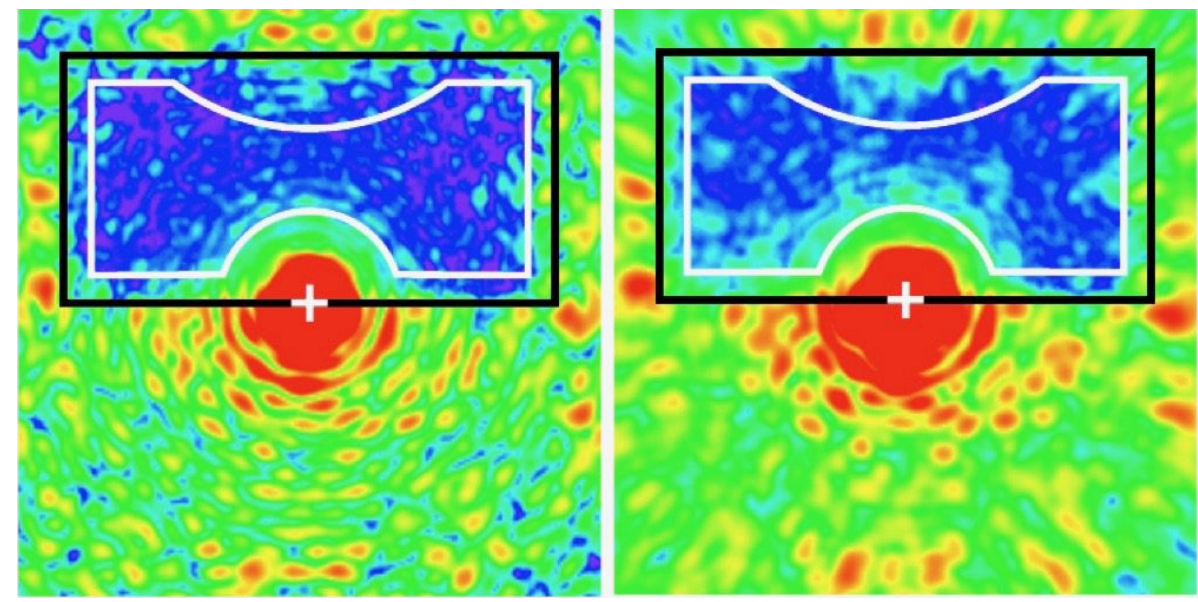

Figure 6. SCC Laboratory results. (Left) Half dark hole $(13 \times 27 \lambda / D)$ obtained in monochromatic (650nm) and (Right) broadband (12,5\% bandwidth in visible) light using the SCC as a focal plane wavefront sensor. The bottom of the dark hole (blue and purple colors) is $10^{-8}$ contrast 47 .

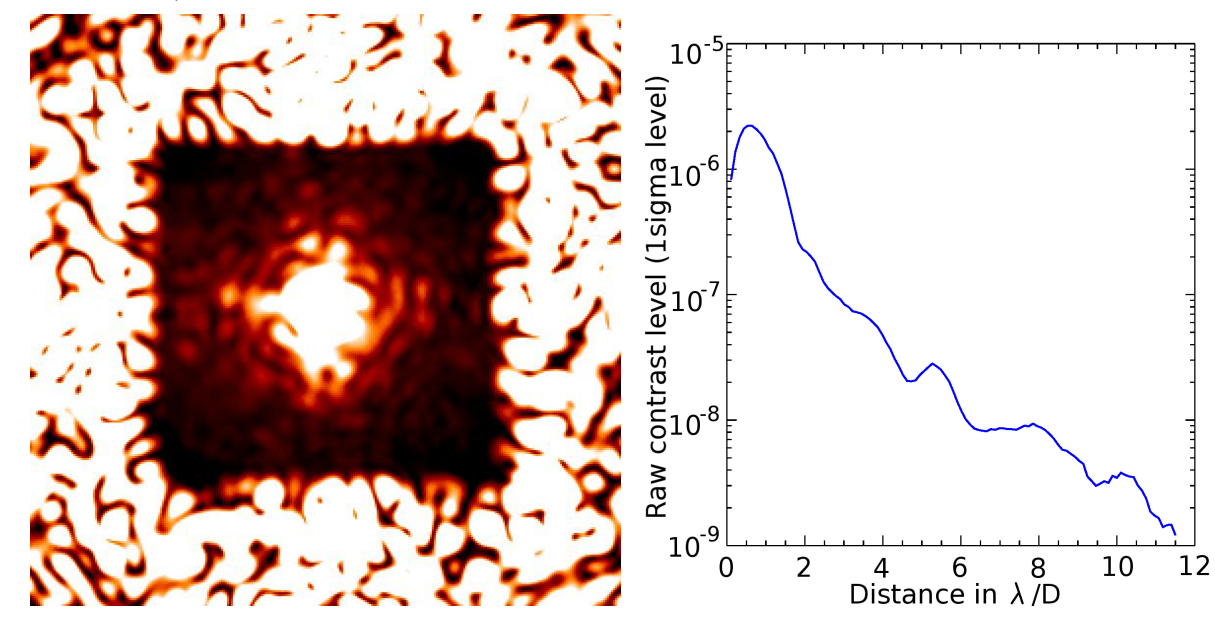

Figure 7. SCC Laboratory results. (Left) Full dark hole $(17 \times 17 \lambda / D)$ obtained in monochromatic light (785nm) using the SCC as a focal plane wavefront sensor and two deformable mirrors. No CDI is applied. (Right) Normalized azimuthal standard deviation associated to the image 50.

\subsection{Pairwise probing}

Pairwise probing uses pairs of DM motions (probes), of the same magnitude but opposite sign, to modulate the electric field in defined regions of the science focal plane. Pairs of probes are used so that sums and differences between images cancel out common-mode terms, such as probe intensity or probe/star cross terms. By combining two or more pairs of probes with an unprobed image and a model of the coronagraphic system, the complex electric field of the starlight and the incoherent residual may be simultaneously estimated across large focalplane areas. In pairwise probing, the incoherent residual includes both terms which are truly incoherent with the starlight, such as planet and exozodiacal light, and estimation error in the coherent signal, which complicates subsequent analysis; the estimation error is generally the dominant term until the coherent level is $3-4 \times$ the incoherent floor.

A common choice for probes is to place a pair of sinc functions on the DM, one oriented along the $\mathrm{x}$-axis and one along the y-axis; these are then multiplied by a sine wave whose phase may be modulated. The sinc phase modulation creates a rectangular region, while the sine makes two copies of this region on either side of the PSF peak. Modulating the sine wave shifts the relative phase of the two rectangles, beating the probe field against 
the remaining speckle field. Further extensions to this method include the use of several probes, which increase the robustness to noise at the expense of overhead, the use of an estimator such as a Kalman filter to replace one of the probes while incorporating previous knowledge about the testbed state, and the use of holographic gratings to introduce probe images into the focal plane concurrently with the science exposure.

For the primary description of the technique, including the mathematical description of the underlying algorithm, see 11. Discussion of the role of estimation error in the incoherent signal can be found in 52, extensions to pairwise probing are covered in 53, and the introduction of holographically generated probes is discussed in 22.

Pairwise probing with sequential DM probes has been demonstrated at several laboratory testbeds, including NASA/JPL, Princeton, and NASA/Ames, with an eye towards space applications. These estimation methods have been combined with correction algorithms such as electric field conjugation (EFC) or stroke minimization to create very-high-contrast dark holes in stabilized environments; estimates of coherent and incoherent components are a byproduct of this correction procedure. A summary of high-contrast results as of 2013 is given in Table 1 of 54; in that table, HCIT (NASA/JPL) and ACE (NASA/Ames) use pairwise probing in their estimation. Most recent results from testing for the WFIRST CGI instrument 55 have yielded $1.6 \times 10^{-9}$ over $3-9 \lambda / D$ in a $10 \%$ band with an hybrid Lyot coronagraph 56 and $1.1 \times 10^{-8}$ in an $18 \%$ band with a shaped pupil coronagraph 57,58 .
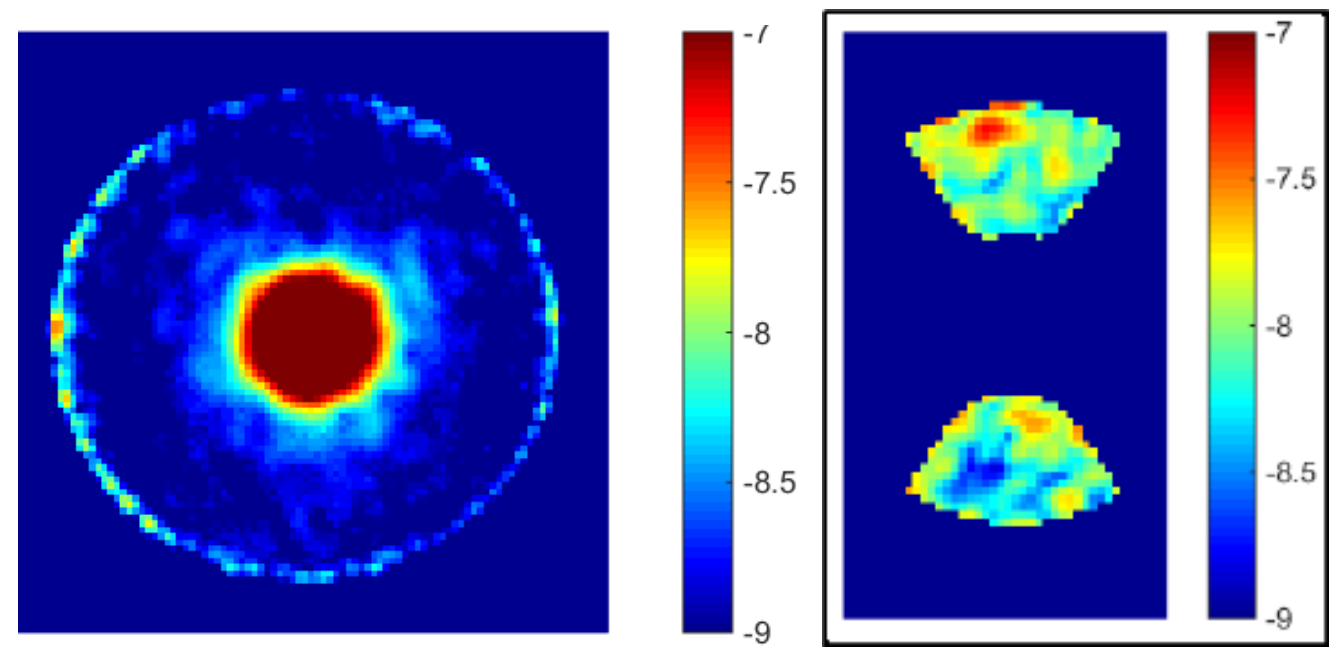

Figure 8. Laboratory results from WFIRST CGI testing at JPL, using pairwise probing for wavefront estimation. Both are shown on the same base-10-log contrast scale. Left. Best narrow-field-of-view result with an HLC coronagraph $\left(1.6 \times 10^{-9}\right.$ contrast $\left.3-9 \lambda / D, 10 \%\right)$ Right. Best spectroscopy-mode result with an SPC coronagraph $\left(1.1 \times 10^{-8}\right.$ contrast $3-9 \lambda / D, 18 \%)$

\subsection{Speckle nulling}

Sinusoidal phase patterns in the pupil plane (which are easily implemented with a DM) correspond to symmetric speckles in the image plane. A small amplitude cosine phase aberration corresponds to symmetric, image plane speckles which are purely imaginary. Likewise, sinusoidal phase aberrations in the pupil plane produce purely real, and antisymmetric speckles in the image plane. Therefore, any speckle in the image plane can be nulled by introducing a sum of sine and cosine phase aberrations in the pupil plane such that the real component of the speckle is nulled by the amplitude of the sine wave, and the imaginary component is nulled by the amplitude of the cosine wave. In practice, since the real and imaginary components of the image plane speckles are unknown, a magnitude and phase of a cosine wave are initially estimated, and applied to the DM. For a given amplitude, the phase of the sinusoidal wave is varied by three steps of $\pi / 2$ phase increments, and the speckles brightness is monitored during these phase steps. From these four intensity measurements, it is possible to estimate the real and imaginary parts or the speckle electric field, and the appropriate anti-speckle is applied to reduce the speckles brightness. The process is repeated until the speckles brightness reaches an acceptable threshold. 
This method is powerful in its simplicity. It relies upon no assumption of the system whatsoever. Typically, the method is used to suppress several speckles simultaneously. Because of its simplicity, it has been used for many high-contrast imaging systems. In order to implement the system, the only requirement is to add a cosine phase ripple of a given amplitude and phase to the DM, and to record the resulting science image. The steps can be implemented on the sky, and will be effective depending upon the magnitude of the speckles, and the sample time of the science camera. Of course a larger number of steps during the phase scan can be used to improve the precision of the sinusoidal fit, but the scan time must be taken into account when considering the timescale of changes in the wavefront. Given that at minimum 4 images are needed with the probes during the phase scan, the maximum science duty cycle (frames that do not have any DM modulation during capture) is $20 \%$ during the nulling process. If one intends to use this loop to chase speckles from turbulence, then this duty cycle is fixed. However, if the goal is to null quasi-static speckles only, then the nulling process can be run for a period and once it has converged, the dark hole could be maintained while science exposures are collected at $100 \%$ duty cycle (no probes applied). Updates to the dark hole need to be administered as the quasi-statics change with time but this will be on minute-10s of minute timescales. Of course, if CDI techniques are employed in post-processing, then it may be possible to utilize all frames (with and without probes) for the final data reduction. This has not been shown to date.

Speckle nulling was first demonstrated in the laboratory on the HCIT testbed at JPL 12. More recently it has been tested on the SCExAO testbed in both the laboratory 59 and on-sky 13. The laboratory tests were even conducted with a PIAA coronagraph to demonstrate that the remapping function did not affect the ability to null the speckles 59. The first on-sky demonstration was limited by the wavefront control provided by the Subaru Telescope facility AO system, AO188 and only a modest improvement of a factor of 2 in the variance was observed over the area of the first Airy ring 13. In more recent work, this method was shown to convincingly suppress quasi-static speckles over the entire control region of the DM (one side of the image) in the presence of atmospheric speckles 27. A similar approach has been tested on Palomar in the $\mathrm{H}$ band and Keck in the $\mathrm{L}$ band 60. The latter included the use of the vortex coronagraph. Unlike in the SCExAO example where the quasi-static speckles were a result of amplitude errors, the Keck demonstration was capable of creating a $360^{\circ}$ dark hole around the PSF. The contrast was greatly improved by applying this technique, but was limited in spatial frequency due to the finite number of actuators on the Keck DM and the slow read out rate of the science camera.

The $\mathrm{H}$ band nulling demonstration on SCExAO was limited by the finite speed and non-negligible read out noise $\left(\sim 120 e^{-}\right)$of the InGaAs camera used, so it could not chase the atmospheric speckles which set an upper limit to the maximum contrast improvement possible with this technique on-sky. New detector technologies like the SAPHIRA and MKIDS will help alleviate these issues. However, this technique is most effective if it can be applied when operating in the diffraction limited regime for which you need to be able to run this in conjunction with a high order AO system. Inroads into offsetting the probe signals from the pyramid WFS correction in the SCExAO system have been made, but significant work still remains in this area.

\subsection{Phase retrieval}

Phase retrieval methods are regularly used in high-contrast imaging testbeds for calibration purposes. For example, phase retrieval can be used to obtain the DM registration required for testbed models to enable the mid-spatial frequency wavefront control loop. Another common usage of phase retrieval on a testbed is to obtain a new DM flat setting that improves the Strehl ratio and can aid in improving the mid-spatial frequency control loop convergence. Thus a phase retrieval process is almost always used as part of the initial testbed model calibration, and it can also be re-run periodically to update the model.

There exist a wide variety of different phase retrieval implementations (these families of methods are classified in 14). The most common is based on a Gerchberg-Saxton iterative process in which a set of focal-plane images are collected. A focal plane image is transformed to a pupil plane, known amplitude and phase constraints are applied before transforming back to the image plane and repeating the process. One problem with the classical Gerchberg-Saxton process is that convergence to the global minimum solution is not guaranteed with convergence

plateauing around a local minimum. To improve solution convergence, it is useful to introduce a known diversity such as a defocus term. This defocus term can be implemented in a testbed by translation of the camera along the 
optical axis or changing the f-number of the focusing optic. Movement of the secondary was used to analyze phase errors on Hubble 61. Phase retrieval is commonly applied as the first step at JPL's HCIT testbed to obtain the DM setting and its registration 62 and reduce model calibration uncertainty errors for WFIRST experiments 63. Phase retrieval can also be used to estimate errors upstream and downstream of a focal plane mask as was done for the EXCEDE experiment 64. At the NASA Ames testbed, recent experiments have shown that the DM can be used to generate diversity for phase retrieval without requiring accurate parametric knowledge of the diversity 65 .

\subsection{Kernel phase}

The kernel phase approach relies on building an accurate relationship between the phase of the Fourier transform of the focal plane image (Fourier phase) and the phase in the pupil 15,16. This can only be done accurately in the small aberration regime and requires a pupil asymmetry, be it phase or amplitude to break the degeneracy so the problem can be inverted and a unique solution found. This is typically achieved by using an asymmetric pupil mask that masks a small portion on one side of the pupil. The full process for sensing and extracting the phase aberration and associated correction can be seen in Fig. 9.

Kernel phase techniques were originally used for imaging to study Brown dwarfs on the Palomar telescope 66. In regards to wavefront control, the technique was experimentally validated on SCExAO in the H-band with the internal source first 16, and then on-sky where it was used to correct the first 10 Zernike modes 67 and more recently to control the island effect (low-wind effect, atmospheric turbulence 68) in closed-loop operation. It operated successfully but was limited by a low temporal bandwidth and low sensitivity, both results of the properties of the detector used.

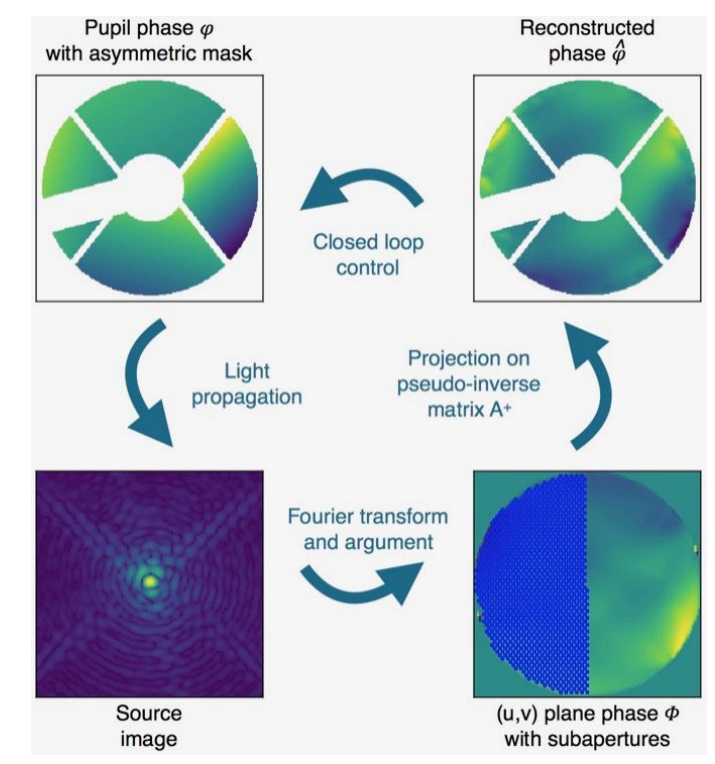

Figure 9. A depiction of the scheme of the phase reconstruction process with an Asymmetric Pupil Fourier Wavefront sensor: (Top Left) phase map $\varphi$ within the asymmetric pupil mask, (Bottom Left) source image on the camera, (Bottom Right) phase $\Phi$ of the Fourier-plane signature in the $(\mathrm{u}, \mathrm{v})$ plane overlapped with half of the subaperture discretization, and (Top Right) the reconstructed pupil phase map $\hat{\varphi}$ from the pseudo-inverse matrix $\mathbf{A}^{+}$. The matrix A links the phase in the Fourier plane and in the pupil plane.

\subsection{Phase shifting interferometry}

A phase shifting interferometer can be envisaged in numerous ways. Bottom et al. demonstrated one possible approach that worked in conjunction with the stellar double coronagraph in the Palm 3k instrument on the Palomar Telescope 17. The two vortex coronagraphs in series sent the rejected star light through the center of a reflective mirror, while the companion light and the residual leaked star light reflected from the mirror and were 
sent to the science camera. By placing a small mirror mounted on a linear translation stage inside the center of the Lyot stop (mirror that separated star from planet light), a small fraction of the rejected light was reflected back into the primary science beam and owing to its small size in the pupil diffracted into a large envelope in the focal plane which encompassed the entire PSF and halo interfering with it. By pistoning the small mirror the envelope was phase shifted with respect to the halo and hence the speckles were modulated in brightness. As the readout times of the detector were slow, the modulation in brightness revealed the quasi-static aberrations rather than speckles due to the turbulence (which evolve much faster). The brightness of the entire speckle halo was monitored simultaneously and CDI techniques were applied in post processing to extract the coherent and incoherent parts of the focal plane image and ultimately resulted in the detection of a known substellar companion 17 .

\subsection{Phase sorting interferometry}

Phase sorting interferometry is a non-invasive technique that can recover both phase and amplitude errors 18 . It uses the residual wavefront errors of the adaptive optics system as phase diversity. The residual wavefront errors in an AO system are continuously measured by the wavefront sensor. The measured wavefront can be propagated analytically through the optical system, yielding the expected speckle halo in the science focal plane, both in phase and amplitude. These complex speckle estimates are then compared to the observed focal-plane images, and an estimate for the static speckle halo is extracted from the interferometric signal between residual AO speckles and unwanted static speckles. This estimate can be propagated back to the pupil plane and corrected by applying a wavefront sensor offset, if closed-loop operation is required. Additionally, an incoherent image can be computed using the same data set 19,69. An overview of the measurement process of phase sorting interferometry is shown in Fig. 10.

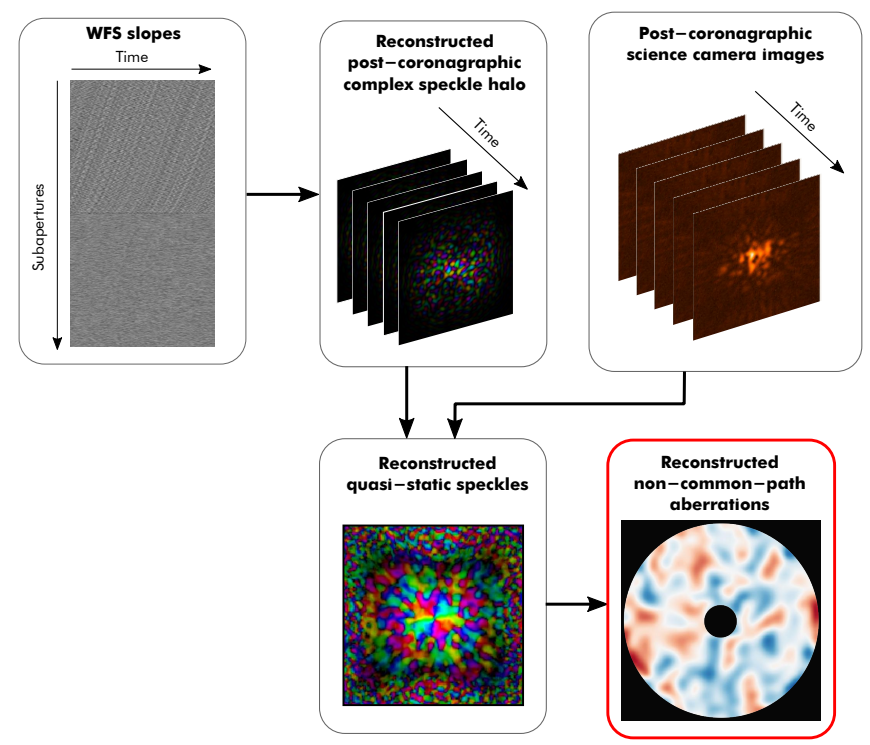

Figure 10. An overview of the measurement process of phase sorting interferometry.

As phase sorting interferometry just requires a continuous stream of wavefront sensor telemetry and highspeed science camera images, and no modulation of the incoming wavefront, it can in principle be included on any existing high-contrast imaging system by only changing the control software. Phase sorting interferometry will be used for closed-loop NCP error correction on the high-contrast imaging arm on ERIS.

\section{LESSONS LEARNED / THINGS TO KEEP IN MIND}

From the forays into common-path WFSing to date, it is possible to suggest a few things to keep in mind when developing these systems. 
1. If the goal is to apply these methods in real-time, the control system (RTC) must be flexible enough to support multiple sensors at real-time rates. Additionally, the availability of AO WFS telemetry is of high-value, to assist NCP WFS techniques and - later down the road - CDI data reduction efforts.

2. The degree to which these sensors are effective depends upon the rate at which the speckles evolve and the rate at which they are sampled. Residual AO speckles have a life-time of approximately the clearing time of the atmospheric phase screen over the input aperture (for a $10 \mathrm{~m}$ telescope, and average wind speed of $10 \mathrm{~m} / \mathrm{s}$, the lifetime is roughly 1 second.) Of course, long-lived non-common path instrumental speckles can have a much longer time constant. Although these appear to be very reasonable time scales, most science cameras have an observing cadence that is much slower. For instance the HAWAII2 has typical full-frame readout times of $1.5 \mathrm{~s}$. The ideal science camera then is one that has low-noise readout with full frame readout rates of a few 10s of Hertz.

3. Coherent sensing, by definition, requires that pathlength differences between the science and the reference (or probe) be long-term stable to within the coherence length $(\lambda *(\lambda / \delta \lambda))$. In particular for phase-modulated reference beams, the zero-point optical path length difference (OPD) must be maintained to within a fraction of a wavelength $(\lambda / 8)$ over the full science observation. This requires careful opto/mechanical engineering.

\section{MILESTONES FOR THE FIELD}

The aim of this section is to outline some goals that would advance the field of common path wavefront sensing/CDI. The ultimate goal is to use the results of tests conducted to address the milestones laid out below in order to drive the design of future ELT and space mission high contrast imaging instruments. The goals outlined are not specific to a testbed or contrast level but are rather guidelines which should be used to direct characterization efforts. The milestones are posed as questions.

- How well do we know the complex field in the region where common-path WFSing/CDI is applied? The ultimate goal here is to show that common-path WFSing techniques can be used to understand the complex field to a level of $90 \%$ (or in other words the difference between our estimate of the incoherent field and the measured field is at the $10 \%$ level). This goal can be broken up in tiers which include both laboratory and on-sky testing/validation. These should be tested in various contrast ranges (i.e. lower contrasts for ground-based applications vs space-based applications). These tests can include standard CDI probing techniques like the introduction of known probe shapes/aberrations for estimation. The generation of incoherent speckles with the DM (modulated faster than the exposure time) may be useful for the ultimate testing of this milestone.

The ultimate demonstration of the successful knowledge of the complex field can be best shown through a direct comparison of the contrast/detectability of a faint companion using standard high-contrast imaging techniques versus CDI techniques.

- How well do we understand our instrument? The goal is to develop a comprehensive model of an instrument and apply common path wavefront sensing/CDI techniques and determine if the experimental results are consistent with the model to within a factor of 2 . This will tell us about any elements that were overlooked in the model, which will inform for future model design for similar systems.

The second aspect of this goal is that once a comprehensive model has been established, it would be informative to study the gain of each common-path WFSing/CDI technique. This should be characterized as a function of: varying amounts of noise (or at least with realistic noise) for both the ground and space-based high contrast imaging cases.

- What are the limits of my common path WFSing technique? Ultimately, we want to understand the relative performance of each common-path WFSing/CDI technique in various regimes of wavefront correction/contrast. For each technique listed in table 1 above, we want to understand the temporal, spectral and spatial bandwidths, science duty cycle and polarization state. 
The results of such characterizations will inform the appropriate parameter space within which each common-path WFSing technique is applicable and support appropriate selection for future ELT and spacebased high contrast instruments.

\section{SUMMARY}

We have demonstrated that there are now many common path wavefront sensing/CDI techniques that are applicable to high contrast imaging. They are in various states of development and have shown differing levels of performance. As the environmental parameters for each testbed and demonstration varied wildly it is hard to make overarching conclusions on the most effective approach at this time, but it is clear that further development and direct comparisons will be needed. These techniques show great promise and will no doubt contribute to imaging extremely faint exoplanets on future GSMTs or space telescopes. We aim to expand this review and submit it as part of a series of publications to JATIS.

\section{ACKNOWLEDGMENTS}

The authors would like to acknowledge the Lorentz Center for hosting and to a large extend funding the Optimal Optical Coronagraph workshop in Leiden 2017. Additional funding for the workshop was provided by the European Research Council under ERC Starting Grant agreement 678194 (FALCONER) granted to Frans Snik. This formed the platform where this work was carried out.

\section{REFERENCES}

[1] Ruane, G. e. a., "Review of high-contrast imaging systems for current and future ground- and space-based telescopes I: coronagraph design methods and optical performance metrics, paper 10698-98," in [Society of Photo-Optical Instrumentation Engineers (SPIE) Conference Series], SPIE (2018).

[2] Snik, F. e. a., "Review of high-contrast imaging systems for current and future ground-based and spacebased telescopes III: technology opportunities and pathways, paper 10706-91," in [Society of Photo-Optical Instrumentation Engineers (SPIE) Conference Series], SPIE (2018).

[3] Wilby, M. J., Keller, C. U., Snik, F., Korkiakoski, V., and Pietrow, A. G. M., "The coronagraphic Modal Wavefront Sensor: a hybrid focal-plane sensor for the high-contrast imaging of circumstellar environments," $A \mathscr{E} A$ 597, A112 (2017).

[4] Guyon, O., Miller, K., Males, J., Belikov, R., and Kern, B., "Spectral Linear Dark Field Control: Stabilizing Deep Contrast for Exoplanet Imaging Using out-of-band Speckle Field," ArXiv e-prints (2017).

[5] Miller, K., Guyon, O., and Males, J., "Spatial linear dark field control: stabilizing deep contrast for exoplanet imaging using bright speckles," Journal of Astronomical Telescopes, Instruments, and Systems 3(4), 049002 (2017).

[6] Ygouf, M., Mugnier, L. M., Mouillet, D., Fusco, T., and Beuzit, J.-L., "Simultaneous exoplanet detection and instrument aberration retrieval in multispectral coronagraphic imaging," $A \xi A$ 551, A138 (2013).

[7] Paul, B., Sauvage, J.-F., Mugnier, L. M., Dohlen, K., Petit, C., Fusco, T., Mouillet, D., Beuzit, J.-L., and Ferrari, M., "Compensation of high-order quasi-static aberrations on SPHERE with the coronagraphic phase diversity (COFFEE)," A\&A 572, A32 (2014).

[8] Mas, M., Baudoz, P., Rousset, G., and Galicher, R., "Tip-tilt estimation and correction using FQPM coronagraphic images," A $\& A$ 539, A126 (2012).

[9] Huby, E., Baudoz, P., Mawet, D., and Absil, O., "Post-coronagraphic tip-tilt sensing for vortex phase masks: The QACITS technique," A\&\&A 584, A74 (2015).

[10] Baudoz, P., Boccaletti, A., Baudrand, J., and Rouan, D., "The Self-Coherent Camera: a new tool for planet detection," in [IAU Colloq. 200: Direct Imaging of Exoplanets: Science Techniques], Aime, C. and Vakili, F., eds., 553-558 (2006).

[11] Give'on, A., Kern, B. D., and Shaklan, S., "Pair-wise, deformable mirror, image plane-based diversity electric field estimation for high contrast coronagraphy," in [Techniques and Instrumentation for Detection of Exoplanets V], SPIE 8151, 815110 (Oct. 2011). 
[12] Borde, P. J., Traub, W. A., Kern, B. D., Trauger, J. T., and Kuhnert, A. C., "Speckle nulling for exoplanet detection with space-based coronagraphic telescopes," in [Society of Photo-Optical Instrumentation Engineers (SPIE) Conference Series], SPIE 6265, 62651C (2006).

[13] Martinache, F., Guyon, O., Jovanovic, N., Clergeon, C., Singh, G., Kudo, T., Currie, T., Thalmann, C., McElwain, M., and Tamura, M., "On-Sky Speckle Nulling Demonstration at Small Angular Separation with SCExAO," PASP 126, 565 (June 2014).

[14] Fienup, J. R., "Phase retrieval algorithms: a comparison," Applied Optics 21, 2758-2769 (1982).

[15] Martinache, F., "Kernel Phase in Fizeau Interferometry," ApJ 724, 464-469 (2010).

[16] Martinache, F., "The Asymmetric Pupil Fourier Wavefront Sensor," PASP 125, 422 (2013).

[17] Bottom, M., Wallace, J. K., Bartos, R. D., Shelton, J. C., and Serabyn, E., "Speckle suppression and companion detection using coherent differential imaging," MNRAS 464, 2937-2951 (Jan. 2017).

[18] Codona, J. L. and Kenworthy, M., "Focal Plane Wavefront Sensing Using Residual Adaptive Optics Speckles," ApJ 767, 100 (Apr. 2013).

[19] Frazin, R. A., "Utilization of the Wavefront Sensor and Short-exposure Images for Simultaneous Estimation of Quasi-static Aberration and Exoplanet Intensity," ApJ 767, 21 (2013).

[20] Codona, J. L., Kenworthy, M. A., Hinz, P. M. and Angel, J. R. P., and Woolf, N. J., "A high-contrast coronagraph for the MMT using phase apodization: design and observations at 5 microns and $2 \lambda / \mathrm{D}$ radius," in [Society of Photo-Optical Instrumentation Engineers (SPIE) Conference Series], SPIE 6269, $62691 \mathrm{~N}$ (June 2006).

[21] Kenworthy, M. A., Codona, J. L., Hinz, P. M. and Angel, J. R. P., Heinze, A., and Sivanandam, S., "First On-Sky High-Contrast Imaging with an Apodizing Phase Plate," ApJ 660, 762-769 (May 2007).

[22] Por, E. H. and Keller, C. U., "Focal-plane electric field sensing with pupil-plane holograms," in [Adaptive Optics Systems V], Proc. SPIE 9909, 990959 (2016).

[23] Haffert, S. Y., Wilby, M. J., Keller, C. U., and Snellen, I. A. G., "The Leiden EXoplanet Instrument (LEXI): a high-contrast high-dispersion spectrograph," in [Ground-based and Airborne Instrumentation for Astronomy VI], SPIE 9908, 990867 (2016).

[24] Wilby, M. J., Keller, C. U., Haffert, S., Korkiakoski, V., Snik, F., and Pietrow, A. G. M., "Designing and testing the coronagraphic Modal Wavefront Sensor: a fast non-common path error sensor for high-contrast imaging," in [Adaptive Optics Systems V], SPIE 9909, 990921 (2016).

[25] Haffert, S. Y., Wilby, M. J., Keller, C. U., Snellen, I., Doelman, D. S., Por, E. H., van Kooten, M., Bos, S. P., and Wardenier, J., "On-sky results of high-dispersion integral-field spectroscopy and high-contrast imaging with the Leiden EXoplanet Instrument(LEXI)," in [Society of Photo-Optical Instrumentation Engineers (SPIE) Conference Series], SPIE 10703 (June 2018).

[26] Groff, T., Chilcote, J., Brandt, T., Kasdin, N. J., Galvin, M., Loomis, C., Rizzo, M., Knapp, G., Guyon, O., Jovanovic, N., Lozi, J., Currie, T., Takato, N., and Hayashi, M., "First light of the CHARIS high-contrast integral-field spectrograph," in [Society of Photo-Optical Instrumentation Engineers (SPIE) Conference Series], Society of Photo-Optical Instrumentation Engineers (SPIE) Conference Series 10400, 1040016 (2017).

[27] Jovanovic, N., Martinache, F., Guyon, O., Clergeon, C., Singh, G., Kudo, T., Garrel, V., Newman, K., Doughty, D., Lozi, J., Males, J., Minowa, Y., Hayano, Y., Takato, N., Morino, J., Kuhn, J., Serabyn, E., Norris, B., Tuthill, P., Schworer, G., Stewart, P., Close, L., Huby, E., Perrin, G., Lacour, S., Gauchet, L., Vievard, S., Murakami, N., Oshiyama, F., Baba, N., Matsuo, T., Nishikawa, J., Tamura, M., Lai, O., Marchis, F., Duchene, G., Kotani, T., and Woillez, J., "The Subaru Coronagraphic Extreme Adaptive Optics System: Enabling High-Contrast Imaging on Solar-System Scales," PASP 127, 890 (Sept. 2015).

[28] Sauvage, J.-F., Mugnier, L. M., Rousset, G., and Fusco, T., "Analytical expression of long-exposure adaptive-optics-corrected coronagraphic image. first application to exoplanet detection," J. Opt. Soc. Am. A 27(11), A157-A170 (2010).

[29] Cantalloube, F., Ygouf, M., Mugnier, L. M. and Mouillet, D., Herscovici-Schiller, O., and Brandner, W., "Status of the MEDUSAE post-processing method to detect circumstellar objects in high-contrast multispectral images," in [AO4ELT Conference - Adaptative Optics for Extremely Large Telescopes proceedings], (2017). 
[30] Ygouf, M., Beichman, C., Hodapp, K., and Roellig, T., "High-contrast imaging with the JWST-NIRSpec Integral Field Unit," in [SF2A-2017: Proceedings of the Annual meeting of the French Society of Astronomy and Astrophysics], Reylé, C., Di Matteo, P., Herpin, F., Lagadec, E., Lançon, A., Meliani, Z., and Royer, F., eds., 325-328 (Dec. 2017).

[31] Soummer, R., Pueyo, L., Sivaramakrishnan, A., and Vanderbei, R. J., "Fast computation of Lyot-style coronagraph propagation," Optics Express 15, 15935 (2007).

[32] Paul, B., Mugnier, L. M., Sauvage, J.-F., Ferrari, M., and Dohlen, K., "High-order myopic coronagraphic phase diversity (COFFEE) for wave-front control in high-contrast imaging systems," Optics Express 21, 31751 (Dec. 2013).

[33] Huby, E., Bottom, M., Femenia, B., Ngo, H., Mawet, D., Serabyn, E., and Absil, O., "On-sky performance of the QACITS pointing control technique with the Keck/NIRC2 vortex coronagraph," $A \mathscr{E} A$ A 600, A46 (2017).

[34] Delorme, J. R., N’Diaye, M., Galicher, R., Dohlen, K., Baudoz, P., Caillat, A., Rousset, G., Soummer, R., and Dupuis, O., "Laboratory validation of the dual-zone phase mask coronagraph in broadband light at the high-contrast imaging THD testbed," A\&A 592, A119 (Aug. 2016).

[35] Galicher, R. and Baudoz, P., "Expected performance of a self-coherent camera," Comptes Rendus Physique 8, 333-339 (Apr. 2007).

[36] Galicher, R., Baudoz, P., and Rousset, G., "Wavefront error correction and Earth-like planet detection by a self-coherent camera in space," A $\& A$ 488, L9-L12 (Sept. 2008).

[37] Baudoz, P., Mas, M., Galicher, R., and Rousset, G., "The Self-Coherent Camera : a focal plane sensor for EPICS ?," in [Adaptative Optics for Extremely Large Telescopes], 05008 (2010).

[38] Baudoz, P., Dorn, R. J., Lizon, J.-L., Fusco, T., Dohlen, K., Charton, J., Beuzit, J.-L., Puget, P., Mouillet, D., Felt, M., Wildi, F., Barrufolo, A., Kasper, M., and Hubin, N., "The differential tip-tilt sensor of SPHERE," in [Ground-based and Airborne Instrumentation for Astronomy III], SPIE 7735, 77355B (2010).

[39] Galicher, R., Baudoz, P., Rousset, G., Totems, J., and Mas, M., "Self-coherent camera as a focal plane wavefront sensor: simulations," Aध̈A 509, A31 (2010).

[40] Mas, M., Baudoz, P., Rousset, G., Galicher, R., and Baudrand, J., "Self-coherent camera: first results of a high-contrast imaging bench in visible light," in [Ground-based and Airborne Instrumentation for Astronomy III], SPIE 7735, 773566 (July 2010).

[41] Baudoz, P., Mazoyer, J., Mas, M., Galicher, R., and Rousset, G., "Dark hole and planet detection: laboratory results using the self-coherent camera," in [Ground-based and Airborne Instrumentation for Astronomy IV], SPIE 8446, 84468C (Sept. 2012).

[42] Mas, M., Baudoz, P., Mazoyer, J., Galicher, R., and Rousset, G., "Experimental results on wavefront correction using the self-coherent camera," in [Ground-based and Airborne Instrumentation for Astronomy IV], SPIE 8446, 844689 (Sept. 2012).

[43] Mazoyer, J., Baudoz, P., Mas, M., Rousset, G., and Galicher, R., "Experimental parametric study of the self-coherent camera," in [Space Telescopes and Instrumentation 2012: Optical, Infrared, and Millimeter Wave], SPIE 8442, 844250 (Sept. 2012).

[44] Mazoyer, J., Baudoz, P., Galicher, R., Mas, M., and Rousset, G., "Estimation and correction of wavefront aberrations using the self-coherent camera: laboratory results," A $\mathscr{G} A \mathbf{5 5 7}$, A9 (2013).

[45] Mazoyer, J., Baudoz, P., Galicher, R., and Rousset, G., "Direct detection of exoplanets in polychromatic light with a Self-coherent camera," in [Proceedings of the Third AO4ELT Conference], Esposito, S. and Fini, L., eds., 97 (Dec. 2013).

[46] Galicher, R., Baudoz, P., Delorme, J. R., Mazoyer, J., Rousset, G., Firminy, J., Boussaha, F., N'Diaye, M., Dohlen, K., and Caillat, A., "High contrast imaging on the THD bench: progress and upgrades," in [Space Telescopes and Instrumentation 2014: Optical, Infrared, and Millimeter Wave], SPIE 9143, 91435A (Aug. 2014).

[47] Delorme, J. R., Galicher, R., Baudoz, P., Rousset, G., Mazoyer, J., and Dupuis, O., "Focal plane wavefront sensor achromatization: The multireference self-coherent camera," A\&A 588, A136 (Apr. 2016).

[48] Baudoz, P., Galicher, R., Patru, F., Dupuis, O., and Thijs, S., "Status and performance of the THD2 bench in multi-deformable mirror configuration," ArXiv e-prints (Jan. 2018). 
[49] Mazoyer, J., Baudoz, P., Galicher, R., and Rousset, G., "High-contrast imaging in polychromatic light with the self-coherent camera," A\&BA 564, L1 (2014).

[50] Baudoz, P., Galicher, R., Potier, A., Dupuis, O., Thijs, S., and Patru, F.

[51] Gerard, B. L., Marois, C., and Galicher, R., "Fast Coherent Differential Imaging on Ground-Based Telescopes using the Self-Coherent Camera," ArXiv e-prints (June 2018).

[52] Cady, E. and Shaklan, S., "Measurements of incoherent light and background structure at exo-Earth detection levels in the High Contrast Imaging Testbed," in [Space Telescopes and Instrumentation 2014: Optical, Infrared, and Millimeter Wave], SPIE 9143, 914338 (Aug. 2014).

[53] Riggs, A. J. E., Kasdin, N. J., and Groff, T. D., "Recursive starlight and bias estimation for high-contrast imaging with an extended Kalman filter," Journal of Astronomical Telescopes, Instruments, and Systems 2(1), 011017 (2016).

[54] Lawson, P. R., Belikov, R., Cash, W., Clampin, M., Glassman, T., Guyon, O., Kasdin, N. J., Kern, B. D., Lyon, R., Mawet, D., Moody, D., Samuele, R., Serabyn, E., Sirbu, D., and Trauger, J., "Survey of experimental results in high-contrast imaging for future exoplanet missions," in [Techniques and Instrumentation for Detection of Exoplanets VI], SPIE 8864, 88641F (Sept. 2013).

[55] Noecker, M. C., Zhao, F., Demers, R., Trauger, J., Guyon, O., and Jeremy Kasdin, N., "Coronagraph instrument for WFIRST-AFTA," Journal of Astronomical Telescopes, Instruments, and Systems 2, 011001 (Jan. 2016).

[56] Seo, B.-J., Cady, E., Gordon, B., Kern, B., Lam, R., Marx, D., Moody, D., Muller, R., Patterson, K., Poberezhskiy, I., Mejia Prada, C., Sidick, E., Shi, F., Trauger, J., and Wilson, D., "Hybrid lyot coronagraph for wfirst: high-contrast broadband testbed demonstration," SPIE 10400, 10400 - 10400 - 21 (2017).

[57] Cady, E., Balasubramanian, K., Gersh-Range, J., Kasdin, N. J., Kern, B., Lam, R., Mejia Prada, C., Moody, D., Patterson, K., Poberezhskiy, I., Riggs, A. J. E., Seo, B.-J., Shi, F., Tang, H., Trauger, J., Zhou, H., and Zimmerman, N., "Shaped pupil coronagraphy for wfirst: high-contrast broadband testbed demonstration," SPIE 10400, 10400 - 10400 - 15 (2017).

[58] Groff, T., C., M., Cady, E., Rizzo, M. J., Mandell, A., Gong, Q., McElwain, M., Zimmerman, N., Saxena, P., and Guyon, O., "Wavefront control methods for high-contrast integral field spectroscopy," SPIE 10400, $10400-10400-7$ (2017).

[59] Martinache, F., Guyon, O., Clergeon, C., and Blain, C., "Speckle Control with a Remapped-Pupil PIAA Coronagraph," PASP 124, 1288 (Dec. 2012).

[60] Bottom, M., Femenia, B., Huby, E., Mawet, D., Dekany, R., Milburn, J., and Serabyn, E., "Speckle nulling wavefront control for Palomar and Keck," in [Adaptive Optics Systems V], SPIE 9909, 990955 (2016).

[61] Krist, J. E. and Burrows, C. J., "Phase-retrieval analysis of pre-and post-repair Hubble Space Telescope images," App. Opt. 34, 4951-4964 (1995).

[62] Zhou, H., Nemati, B., Krist, J., Cady, E., Kern, B., and Poberezhskiy, I., "Wavefront Control Performance Modeling with wFIRST Shaped Pupil Coronagraph Testbed," in [Society of Photo-Optical Instrumentation Engineers (SPIE) Conference Series], Society of Photo-Optical Instrumentation Engineers (SPIE) Conference Series 10400, 1040005 (2017).

[63] Marx, D., Seo, B.-J., Kern, B., Sidick, E., Nemati, B., and Poberezhskiy, I., "Electric field conjugation in the presence of model uncertainty," in [Society of Photo-Optical Instrumentation Engineers (SPIE) Conference Series], Society of Photo-Optical Instrumentation Engineers (SPIE) Conference Series 10400, 104000P (2017).

[64] Sirbu, D., Thomas, S., Belikov, R., Lozi, J., Bendek, E., Pluzhnik, E., Lynch, D., Zell, P., Guyon, O., and Schneider, G., "Demonstration of broadband contrast at 1.2 $\lambda / \mathrm{D}$ and greater for the EXCEDE Starlight Suppression System," Journal of Astronomical Telescopes, Instruments, and Systems 2(2), 025002 (2016).

[65] Pluzhnik, E., Sirbu, D., Belikov, R., Bendek, E., and Dudinov, V. N., "Wavefront retrieval through random pupil plane phase probes: Gerchberg-Saxton approach," ArXiv e-prints (Sept. 2017).

[66] Pope, B., Martinache, F., and Tuthill, P., "Dancing in the Dark: New Brown Dwarf Binaries from Kernel Phase Interferometry," ApJ 767, 110 (Apr. 2013).

[67] Martinache, F., Jovanovic, N., and Guyon, O., "Closed-loop focal plane wavefront control with the SCExAO instrument," $A \& A$ 593, A33 (2016). 
[68] N'Diaye, M., Martinache, F., Jovanovic, N., Lozi, J., Guyon, O., Norris, B., Ceau, A., and Mary, D., "Calibration of the island effect: Experimental validation of closed-loop focal plane wavefront control on Subaru/SCExAO," A $E A$ 610, A18 (2018).

[69] Por, E. H. and Kenworthy, M. A., "Imaging exoplanets using phase sorting interferometry and coherent differential imaging," in [SPIE ], 10703 (June 2018). 